\title{
Unitary equivalence between the Green's function and Schrödinger approaches for quantum graphs
}

\author{
Fabiano M. Andrade ${ }^{1, *}$ and Simone Severini ${ }^{2,3}$ \\ ${ }^{1}$ Departamento de Matemática e Estatística, Universidade Estadual de Ponta Grossa, 84030-900 Ponta Grossa-PR, Brazil \\ ${ }^{2}$ Department of Computer Science, University College London, London WC1E 6BT, United Kingdom \\ ${ }^{3}$ Institute of Natural Sciences, Shanghai Jiao Tong University, Shanghai 200240, China
}

(Received 3 August 2018; published 6 December 2018)

\begin{abstract}
In a previous work [Andrade et al., Phys. Rep. 647, 1 (2016)], it was shown that the exact Green's function (GF) for an arbitrarily large (although finite) quantum graph is given as a sum over scattering paths, where local quantum effects are taken into account through the reflection and transmission scattering amplitudes. To deal with general graphs, two simplifying procedures were developed: regrouping of paths into families of paths and the separation of a large graph into subgraphs. However, for less symmetrical graphs with complicated topologies as, for instance, random graphs, it can become cumbersome to choose the subgraphs and the families of paths. In this work, an even more general procedure to construct the energy domain GF for a quantum graph based on its adjacency matrix is presented. This new construction allows us to obtain the secular determinant, unraveling a unitary equivalence between the scattering Schrödinger approach and the Green's function approach. It also enables us to write a trace formula based on the Green's function approach. The present construction has the advantage that it can be applied directly for any graph, going from regular to random topologies.
\end{abstract}

DOI: 10.1103/PhysRevA.98.062107

The past decade witnessed a notable interest in the interplay between quantum mechanics and graphs. The area is very rich because its objectives go from tests in spin chains as nanodevices to the explanation of natural phenomena as energy transfer in biological systems. General methods to deal with graphs are always very welcome because the myriad of different topologies make it difficult to develop a unique method that holds for all graphs. In the context of quantum graphs [1-6], a Green's function (GF) approach was first proposed in [7] and explored in depth in [8]. In the latter, to handle general quantum graphs of different topologies, two simplification procedures were developed: (i) the regrouping of infinite many scattering paths into finite families of paths (FP) and (ii) the division of the graph into subgraphs, then solving each subgraph individually by calculating effective scattering amplitudes, and then connecting all the pieces altogether. As described in [8], the GF construction based on these two procedures is very general and useful. However, for large graphs, less symmetrical graphs, graphs that change the connections by some mechanism, or random graphs, it may become really difficult to choose the subgraphs and to define the FP. Furthermore, although the final result is totally independent of the choices of the FP, this choice is not unique, preventing, for instance, the development of a general algorithm for the GF construction.

In this paper, we aim to give an even more general and powerful method for the GF construction for quantum graphs. We shall show that the GF approach (GFA) presented here provides an alternative derivation for the secular determinant, unraveling a unitary equivalence between the GFA and the scattering Schrödinger approach (SSA) [9-11]. Moreover,

\footnotetext{
*fmandrade@uepg.br
}

it also provides another way to derive a trace formula for quantum graphs $[3-5,12,13]$.

A graph $X(V, E)$ is defined as a pair consisting of a set of vertices $V(X)=\{1, \ldots, n\}$ and a set of edges $E(X)=$ $\left\{e_{1}, \ldots, e_{l}\right\}$, where each edge is a pair of vertices [14]. The graph topology is described in terms of the adjacency matrix $A(X)$ of dimension $n \times n$, where the $i j$ th element $A_{i j}(X)$ is 1 if $\{i, j\} \in E(X)$ and zero otherwise. Two vertices are neighbors whether they are connected by an edge. The set $E_{i}=\{j:\{i, j\} \in E(X)\}$ is the neighborhood of the vertex $i \in V(X)$. We denote by $E_{i}^{k}=E_{i} \backslash\{k\}$ the set of neighbors of the vertex $i$, but with the vertex $k$ excluded. The degree of $i$ is $d_{i}=\left|E_{i}\right|=\sum_{j=1}^{n} A_{i j}(X)$. These definitions refer to discrete graphs. To discuss quantum graphs, it is necessary to equip the graphs with a metric. A metric graph $\Gamma(V, E)$ is a graph in which is assigned a positive length $\ell_{e_{s}} \in(0,+\infty)$ to each edge, thus defining the set $\ell=\left\{\ell_{e_{1}}, \ldots, \ell_{e_{l}}\right\}$. When a single ended edge $e_{s}$ is taken as semi-infinite $\left(\ell_{e_{s}}=+\infty\right)$, it is called a "lead." A quantum graph is a metric graph in which it is possible to define a Schrödinger operator along with appropriated boundary conditions (BCs) at the vertices or, more formally, a triple $\{\Gamma(V, E), H$, bc $\}$ with $H$ a differential operator and bc a set of BCs. For the free Schrödinger operator $H=-\left(\hbar^{2} / 2 m\right) d^{2} / d x^{2}$ it leads us to the eigenvalue equation

$$
-\psi_{\{i, j\}}^{\prime \prime}(x)=k^{2} \psi_{\{i, j\}}(x),
$$

where $k=\sqrt{2 m E / \hbar^{2}}, m$ is the mass, $E$ is the energy, and $\psi_{\{i, j\}}$ is the wave function on the edge $\{i, j\}$. Hereafter we consider just simple connected graphs.

An important ingredient in the GFA for quantum graphs is the individual scattering amplitudes defined at each one of the graph vertices, in such a way that we can define a scattering matrix $\sigma_{j}$ for each vertex $j$ of the graph. The 


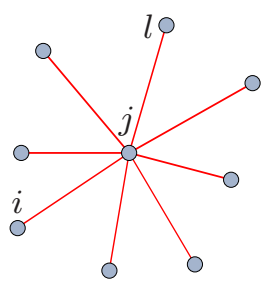

FIG. 1. Locally, any graph looks like a star graph.

scattering amplitudes are entirely determined by the BCs defined at each vertex and the most general ones, which are consistent with quantum flux conservation and fulfill the required condition of self-adjointness, were discussed in [15]. Without loss of generality, in an arbitrary graph locally we can always treat a vertex $j$ with its edges as a star graph. A star graph on $n$ vertices, $S_{n}$, is a graph where one central vertex has degree $n-1$ and all others vertices have degree 1. Consider thus a star graph as the one depicted in Fig. 1 and let $\Psi(j)=\left(\psi_{\{j, 1\}}(j), \ldots, \psi_{\{j, n\}}(j)\right)^{T}$. The most general BCs that are consistent with the self-adjoint condition [8] are totally defined by two $d_{j} \times d_{j}$ matrices $\mathcal{A}_{j}$ and $\mathcal{B}_{j}$ such that [15]

$$
\mathcal{A}_{j} \Psi(j)+\mathcal{B}_{j} \Psi^{\prime}(j)=0,
$$

the matrix $\mathcal{A B}^{*}$ is self-adjoint, and the $d_{j} \times 2 d_{j}$ matrix $\left(\mathcal{A}_{j}, \mathcal{B}_{j}\right)$ has the maximal rank $d_{j}$. The scattering amplitudes associated with the $\mathrm{BC}(2)$, can be determined by considering a plane wave on the edge $\{i, j\}$ incident on the vertex $j$ with degree $d_{j}$. Thus the scattering solutions that satisfy the eigenvalue equation (1) are given by

$$
\begin{aligned}
& \psi_{\{i, j\}}(x)=e^{-i k x}+\sigma_{j}^{[\{j, i\},\{i, j\}]}(k) e^{i k x}, \\
& \psi_{\{j, l\}}(x)=\sigma_{j}^{[\{j, l\},\{i, j\}]}(k) e^{i k x} .
\end{aligned}
$$

The quantities $\quad \sigma_{j}^{[\{j, i\},\{i, j\}]}(k)=r_{j}^{[\{j, i\},\{i, j\}]}(k) \quad$ and $\sigma_{j}^{[\{j, p\},\{i, j\}]}(k)=t_{j}^{[\{j, p\},\{i, j\}]}(k)$ are the reflection and transmission amplitudes at the vertex $j$, respectively. By applying the $\mathrm{BC}(2)$, we have

$$
\sigma_{j}(k)=-\left(\mathcal{A}_{j}+i k \mathcal{B}_{j}\right)^{-1}\left(\mathcal{A}_{j}-i k \mathcal{B}_{j}\right) .
$$

So, we can observe that the $\sigma_{j}$ generally depends on $k$ in a nontrivial manner. However, there are certain BCs that are independent of $k$, as, for instance, the case of Dirichlet, Neumann, and Kirchoff BCs [12]. Thus we can see that for quantum graphs it is totally equivalent to set either the $\mathrm{BC}$ or to specify the scattering matrix at the vertex $j$ [15]. As said above, the $\sigma_{j}(k)$ must satisfy the requirement of quantum flux conservation, so it demands that the $\sigma_{j}(k)$ must be unitary, $\boldsymbol{\sigma}_{j}(k) \boldsymbol{\sigma}_{j}^{\dagger}(k)=\mathbb{1}$, and $\boldsymbol{\sigma}_{j}(k)=\boldsymbol{\sigma}_{j}^{\dagger}(-k)$, leading to

$$
\begin{array}{r}
\sigma_{j}^{[\{j, l\},\{i, j\}]}(k)=\left[\sigma_{j}^{[\{i, j\},\{j, l\}]}(-k)\right]^{*}, \\
\sum_{i \in E_{j}} \sigma_{j}^{[\{j, l\},\{i, j\}]}(k)\left[\sigma_{j}^{[\{j, m\},\{i, j\}]}(k)\right]^{*}=\delta_{l m}, \\
\sum_{i \in E_{j}} \sigma_{j}^{[\{i, j\},\{j, l\}]}(k)\left[\sigma_{j}^{[\{i, j\},\{j, m\}]}(k)\right]^{*}=\delta_{l m},
\end{array}
$$

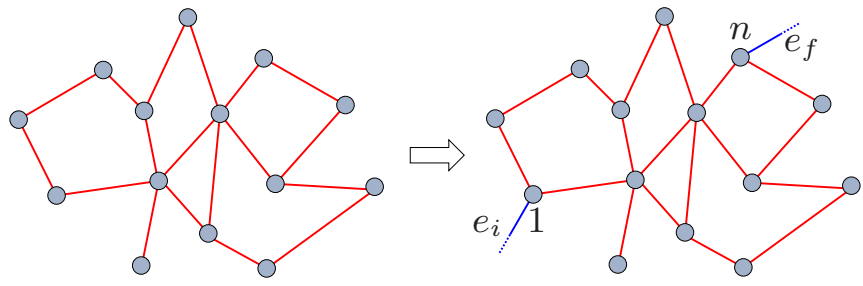

FIG. 2. Graph with two leads added turning it into an open graph.

which are natural generalizations of the usual relations for the scattering amplitudes in 1D scattering problems [16].

Consider a quantum graph $\{\Gamma(V, E), H, \mathbf{b c}\}$ with the adjacency matrix $A(\Gamma)$. Then, add two leads $e_{i}$ and $e_{f}$ to the vertices 1 and $n$, respectively, as shown in Fig. 2, turning it into an open quantum graph, suitable for studying scattering problems. The exact scattering GF for a particle of fixed energy $E=\hbar^{2} k^{2} / 2 m$, with initial position $x_{i}$ in the lead $e_{i}$ and final position $x_{f}$ in the lead $e_{f}$, is given by a sum over all the scattering paths (SP) connecting the points $x_{i}$ and $x_{f}$, where each path is weighted by the product of the scattering amplitudes gained along the path. These scattering amplitudes are determined through the BCs defined at the vertices. Thus the exact scattering GF is written as $[13,17]$ (see also Ref. [8])

$$
G\left(x_{f}, x_{i} ; k\right)=\frac{m}{i \hbar^{2} k} \sum_{\mathrm{SP}} W_{\mathrm{SP}} e^{\left[\frac{i}{\hbar} S_{\mathrm{SP}}\left(x_{f}, x_{i} ; k\right)\right]},
$$

where, for each SP, $S_{\mathrm{SP}}=k L_{\mathrm{SP}}$ is the classical-like action, with $L_{\mathrm{SP}}$ the total path length. The term $W_{\mathrm{SP}}$ is the SP quantum amplitude, constructed from the product of all quantum amplitudes $\sigma_{j}$ acquired along the SP.

Our first goal is to rewrite the GF in a way that it is dependent on of the underlying graph. This will be achieved by using the adjacency matrix of the graph and the following rules: (i) for every vertex $j$ of the graph we define a scattering matrix $\sigma_{j}(k)$ associated with the $\mathrm{BC}$ used at the vertex $j$, (ii) the free propagation along the edge between two vertices $i$ and $j$ contributes with the term $z_{i j}=z_{j i}=e^{i k \ell_{i j}}$, where $\ell_{i j}$ is the length of the edge, and (iii) in each edge between the vertices $i$ and $j$ we define two FP, one going from $i$ to $j$ and another in the reverse direction. They are given by

$$
p_{i j}=\sum_{l \in E_{j}^{n}} z_{i j} \sigma_{j}^{[\{j, l\},\{i, j\}]} A_{j l} p_{j l}+\delta_{j n} z_{i n} \sigma_{n}^{\left[e_{f},\{i, n\}\right]},
$$

and the family $p_{j i}$ is given by the same expression above, but with the swapping of indices $i$ and $j$. Then, in each vertex $i$ we associated one $p_{i j}$ for every $j \in E_{i}$. The last term in (7) is the transmission amplitude at the vertex $n$ from the edge $\{i, n\}$ to the lead $e_{f}$. So, using the above rules, the exact scattering GF for a quantum graph with adjacency matrix $A(\Gamma)$ can be written as

$$
G_{\Gamma}=\frac{m}{i \hbar^{2} k} T_{\Gamma} e^{i k\left(x_{i}+x_{f}\right)},
$$

where $T_{\Gamma}=\sum_{j \in E_{i}} \sigma_{i}^{\left[\{i, j\}, e_{i}\right]} A_{i j} p_{i j}$. Thus we observe that, by employing the adjacency matrix of the graph, we were able to replace an infinite sum over SP by a finite sum over FP in a unique way (except for the possible permutations of the adjacency matrix of the graph). The number of FP is always 
finite. For instance, in the fully connected simple graph on $n$ vertices, $K_{n}$, the number of different FP is twice the number of edges, $2\left(\begin{array}{l}n \\ 2\end{array}\right)$. We can use the Schur-Hadamard product [18] to know which FP need to be considered in a specific graph, $P_{\Gamma}=P \circ A(\Gamma)$, where $P=\left(p_{i j}\right)$ is an $n \times n$ matrix. The main diagonal elements of $P_{\Gamma}$ are zero because no vertex is connected to itself in simple graphs. The FP altogether form a system of equations whose solution provides the exact energy-dependent GF. Once having obtained the exact GF, we have all the possible information from a quantum system [19]. For instance, we can calculate the transmission probability for transverse the graph as a function of the energy of the incident particle, which can be used, for example, to study the presence of resonances [20]. Indeed, $\left|T_{\Gamma}\right|^{2}$ represents the global transmission probability from the lead $e_{i}$ to the lead $e_{f}$ and it is constructed from the individual quantum amplitudes. This kind of construction was already explored, although using a different approach, in [21,22]. Bound-state energies can be obtained from the poles of the GF and the associated wave functions from the respective residues [8].

The construction presented so far is for general quantum graphs. Given the fact that star graphs can be employed as building blocks for larger graphs [10], let us focus on the problem of a quantum star graph, $S_{n}$. Additionally, in order to simplify the notation, here and henceforth, we drop the edge labels from the scattering amplitudes and just use $r(t)$ for the reflection (transmission). To prove the unitary equivalence between the SSA and the GFA for quantum graphs, we start with the SSA by writing the general solutions for the eigenvalue equation (1) on the edges of the $S_{n}$ :

$$
\psi_{\{1, i\}}(x)=a_{1 i} e^{i k x}+b_{1 i} z_{1 i} e^{-i k x},
$$

$\forall i \in E_{1}$, where $a_{1 i}$ and $b_{1 i}$ are ( $k$ dependent) complex amplitudes (we label the central vertex as 1). By applying the BC (2) on the vertices of the quantum star graph, we find

$$
U_{S_{n}}^{S}(k) \mathbf{a}_{S_{n}}=\mathbf{a}_{S_{n}},
$$

where $\mathbf{a}_{S_{n}}=\left(a_{12}, b_{12}, \ldots, a_{1 n}, b_{1 n}\right)^{T}$, and the $2(n-1) \times$ $2(n-1)$ matrix $U_{S_{n}}^{S}(k)$ can be written as a product of two matrices,

$$
U_{S_{n}}^{S}(k)=S_{S_{n}}(k) D_{S_{n}}(k),
$$

with $D_{S_{n}}(k)=\operatorname{diag}\left(z_{12}, z_{12}, z_{13}, z_{13}, \ldots, z_{1 n}, z_{1 n}\right)$ and

$$
S_{S_{n}}(k)=\left(\begin{array}{ccccccc}
0 & r_{2} & 0 & 0 & \ldots & 0 & 0 \\
r_{1} & 0 & t_{1} & 0 & \ldots & t_{1} & 0 \\
0 & 0 & 0 & r_{3} & \ldots & 0 & 0 \\
t_{1} & 0 & r_{1} & 0 & \ldots & t_{1} & 0 \\
\vdots & \vdots & \vdots & \vdots & \ddots & \vdots & \vdots \\
0 & 0 & 0 & 0 & \ldots & 0 & r_{n} \\
t_{1} & 0 & t_{1} & 0 & \ldots & r_{1} & 0
\end{array}\right) .
$$

The scattering amplitudes $r_{i}$, for $i \in E_{1}$, are given by (4) with $d_{i}=1$, while $r_{1}$ and $t_{1}$ are given by (4) with $d_{1}=n-1$. The edge propagation matrix $D_{S_{n}}(k)$ has the metric information of the quantum star graph and the scattering matrix $S_{S_{n}}(k)$ has the information of the scattering process at the vertices. From the relations for the scattering amplitudes in (5), it follows that $S_{S_{n}}(k)$ is unitary, and the unitarity of $D_{S_{n}}(k)$ is direct. Thus $U_{S_{n}}^{S}(k)$ is also unitary and it is referred to as

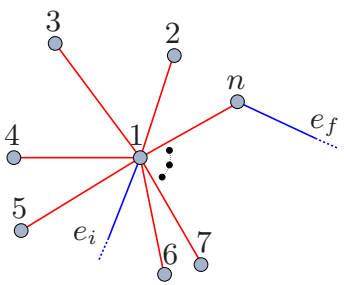

FIG. 3. Star graph on $n$ vertices with two leads added turning it into an open star graph.

the quantum evolution map [23]. The action of this map is a composition of a propagation along the edges followed by a scattering process at the vertices. The system (10) has a nontrivial solution for the wave number $k>0$, when

$$
\zeta_{S_{n}}^{S}(k):=\operatorname{det}\left[\mathbb{1}-U_{S_{n}}^{S}(k)\right]=0,
$$

which is the secular determinant and whose zeros define the quantum star graph spectra.

Now, consider the quantum star graph when we attach a lead $e_{i}$ to the vertex 1 and a lead $e_{f}$ to the vertex $n$ [24] as depicted in Fig. 3. Thus the scattering GF is

$$
G_{S_{n}}=\frac{m}{i \hbar^{2} k} T_{S_{n}} e^{i k\left(x_{i}+x_{f}\right)}
$$

with $T_{S_{n}}=\sum_{j \in E_{1}} t_{1} p_{1 j}$, where the families

$$
\begin{aligned}
& p_{1 j}=z_{1 j}\left(r_{1} p_{j 1}+\delta_{j n} t_{n}\right), \\
& p_{j 1}=z_{1 j}\left(r_{1} p_{1 j}+\sum_{i \in E_{1}^{1}} t_{1} p_{1 i}\right),
\end{aligned}
$$

form a system of $2(n-1)$ equations. To compare with the SSA, we need to consider bound states. This is accomplished by excluding the transmission at the vertex $n$ to the lead $e_{f}$. In this case, we can write (15) as

$$
U_{S_{n}}^{G}(k) \mathbf{p}_{S_{n}}=\mathbf{p}_{S_{n}},
$$

where $\mathbf{p}_{S_{n}}=\left(p_{12}, p_{21}, \ldots, p_{1 n}, p_{n 1}\right)^{T}$ and (16) has a nontrivial solution for $k>0$ if

$$
\zeta_{S_{n}}^{G}(k):=\operatorname{det}\left[\mathbb{1}-U_{S_{n}}^{G}(k)\right]=0 .
$$

Surprisingly, $U_{S_{n}}^{G}(k)$ can also be factored as a product of $S_{S_{n}}(k)$ and $D_{S_{n}}(k)$, but in opposite order,

$$
U_{S_{n}}^{G}(k)=D_{S_{n}}(k) S_{S_{n}}(k) .
$$

Thus it is also unitary. In fact, $U_{S_{n}}^{G}(k)$ is the quantum evolution map, but now obtained from the GFA. The action of $U_{S_{n}}^{G}(k)$ is a composition of a scattering at the vertices followed by a propagation along the edges. We have the following result for the eigenvalues of $U_{S_{n}}^{S}(k)$ and $U_{S_{n}}^{G}(k)$.

Theorem 1: All the eigenvalues of the quantum evolution maps $U_{S_{n}}^{S}(k)$ and $U_{S_{n}}^{G}(k)$ are identical including the degeneracy.

Proof. Let $\mathbf{a}_{S_{n}}^{\lambda}$ be an eigenvector of the map $U_{S_{n}}^{S}(k)$ with a nonzero eigenvalue $\lambda, U_{S_{n}}^{S}(k) \mathbf{a}_{S_{n}}^{\lambda}=\lambda \mathbf{a}_{S_{n}}^{\lambda}$. Given that $U_{S_{n}}^{S}(k)$ is a unitary map, all its eigenvalues are nonzero and have modulus 1. Multiplying $D_{S_{n}}(k)$ on the left, we 
have $U_{S_{n}}^{G}(k)\left[D_{S_{n}}(k) \mathbf{a}_{S_{n}}^{\lambda}\right]=\lambda\left[D_{S_{n}}(k) \mathbf{a}_{S_{n}}^{\lambda}\right] . D_{S_{n}}(k)$ being unitary, $D_{S_{n}}(k) \mathbf{a}_{S_{n}}^{\lambda}$ is a nonzero eigenvector of $U_{S_{n}}^{G}(k)$ with eigenvalue $\lambda$. To complete our proof, we just reverse our reasoning for $U_{S_{n}}^{G}(k)$. The identical degeneracy of $U_{S_{n}}^{S}(k)$ and $U_{S_{n}}^{G}(k)$ is due to the fact that the unitary operators $S_{S_{n}}(k)$ and $D_{S_{n}}(k)$ preserve the orthogonality of the eigenvectors with the same eigenvalue.

We then conclude that the secular determinants (13) and (17) are equal, thus providing the same spectra. The result above brings us to the following interesting and useful result.

Corollary 1.1: The eigenvector $\mathbf{a}_{S_{n}}^{\lambda}$ with eigenvalue $\lambda$, which are associated with the wave function amplitudes, Eq. (9), can be obtained from the eigenvector $\mathbf{p}_{S_{n}}^{\lambda}$ by $\mathbf{a}_{S_{n}}=$ $S_{S_{n}}(k) \mathbf{p}_{S_{n}}^{\lambda}$, up to an arbitrary phase factor.

So, the wave functions for the quantum star graph can be obtained from the GFA directly from $\mathbf{p}_{S_{n}}^{\lambda}$, without the need to resort to the calculation of the residues of the GF [8]. We can now state our main result about the connection between the maps $U_{S_{n}}^{S}(k)$ and $U_{S_{n}}^{G}(k)$.

Claim 1: The quantum evolution maps $U_{S_{n}}^{S}(k)$ and $U_{S_{n}}^{G}(k)$ are unitarily similar.

Given the properties of these maps, there are strong reasons to believe that this claim works for every $n$. Although a proof for a general $n$ is not known, we checked this for $n=2,3,4,5$ by using Specht's theorem [25]. This theorem provides a necessary and sufficient condition to prove that two matrices are unitarily similar. A word $w(s, t)$ is any finite formal product of nonnegative powers of $s$ and $t$, $w(s, t)=s^{m_{1}} t^{n_{1}} s^{m_{2}} s^{n_{2}} \ldots s^{m_{k}} s^{n_{k}}$, with $m_{1}, n_{1}, \ldots, m_{k}, n_{k} \geqslant$ 0 . The length of the word $w(s, t)$ is the nonnegative integer given by the sum of all exponents in the word, $\sum_{i=1}^{k}\left(m_{i}+n_{i}\right)$.

Theorem 2 (Specht's theorem [25,26]): Two $n \times n$ complex matrices $A$ and $B$ are unitarily similar if and only if

$$
\operatorname{tr} w\left(A, A^{*}\right)=\operatorname{tr} w\left(B, B^{*}\right)
$$

for every word $w(s, t)$ in two noncommuting variables whose length is at most [27]

$$
n \sqrt{\frac{2 n^{2}}{n-1}+\frac{1}{4}}+\frac{n}{2}-2 .
$$

Given the fact that the GF is obtained from the solution of the system of equations in (15), its final form has an important contribution from the secular determinant. In fact, the GF for a quantum star graph on $n$ vertices is seen to be

$$
G_{S_{n}}=\frac{m}{i \hbar^{2} k} \frac{1}{g_{S_{n}}} \prod_{i \in E_{1}}\left(g_{1 i}+r_{i} t_{1} z_{1 i}^{2}\right) t_{1} t_{n} z_{1 n} e^{i k\left(x_{i}+x_{f}\right)},
$$

where $g_{1 i}=1-r_{1} r_{i} z_{1 i}^{2}$ and $g_{S_{n}}$ is the secular determinant in (17). So, the eigenvalues are the poles of the GF and these poles are just the zeros of the secular determinant. Thus the secular determinant for a quantum star graph on $n$ vertices, with general boundary conditions, is obtained directly from Eq. (16). Moreover, the poles have contribution from the classical periodic orbits of the graph. We can exemplify this with the quantum star graph $S_{3}$, for which $g_{S_{3}}=(1-$ $\left.r_{1} r_{2} z_{12}^{2}\right)\left(1-r_{1} r_{3} z_{13}^{2}\right)-r_{2} r_{3} t_{1}^{2} z_{12}^{2} z_{13}^{2}$, and it is possible to see

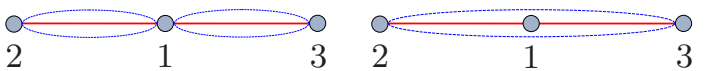

FIG. 4. Periodic orbits for the quantum star graph $S_{3}$.

the contribution of three periodic orbits: one confined in the edge $\{1,2\}$, another one confined in the edge $\{1,3\}$, and the last one that covers the entire graph (see Fig. 4).

Finally, we can write a trace formula from the GFA by considering the secular determinant (17). The spectral counting function $N(k)$ is given by [4,5]

$$
N(k)=\bar{N}(k)+\frac{1}{\pi} \operatorname{Im} \sum_{\nu=1}^{\infty} \frac{1}{v} \operatorname{tr}\left[U_{S_{n}}^{G}(k)\right]^{v},
$$

where $\bar{N}(k)$ corresponds to the smooth part of the counting function and the second term is the oscillatory part. Since the main diagonal of $U_{S_{n}}^{G}(k)$ is zero, $\operatorname{tr}\left[U_{S_{n}}^{G}(k)\right]^{2 v+1}=0$ and $\operatorname{tr}\left[U_{S_{n}}^{G}(k)\right]^{2 v}=2 \sum_{p \in \mathcal{P}_{v}} W_{p} e^{i k \ell_{p}}$, where $W_{p}$ is the product of quantum amplitudes along the periodic orbit, $\ell_{p}$ is the length of the periodic orbit, and $\mathcal{P}_{v}$ is the set of periodic orbits of the graph. For the Neumann BC, $\bar{N}=k \mathcal{L} / 2 \pi+1 / 2$ [5], where $\mathcal{L}=2 \sum_{E} \ell_{i j}$, and we can write the density of states $d(k)=d N(k) / d k$ as

$$
d(k)=\frac{\mathcal{L}}{2 \pi}+\operatorname{Im} \frac{\ell_{p}}{\pi} \sum_{v=1}^{\infty} \frac{1}{v} \sum_{p \in \mathcal{P}_{v}} W_{p} e^{i k \ell_{p}} .
$$

In summary, we have introduced a general and powerful approach for the construction of the GF for quantum graphs based on the adjacency matrices of the graphs. This provides another way to obtain the secular determinant, unraveling a unitary equivalence between the SSA and GFA. An advantage of the GFA is that the system that leads to the secular determinant is obtained in a very direct manner and for general energy-dependent scattering amplitudes (general $\mathrm{BCs}$ ). It also provides us a connection between the poles of the GF and the secular determinant, and enables us to write a trace formula for quantum graphs from the GFA. Moreover, our approach can be used to study quantum walks in graphs with complicated topologies. This subject was studied by one of us in simple topologies in Ref. [28]. Furthermore, for dressed quantum graphs $[29,30]$, i.e., when there are potentials $u_{i j}$ along the edges, our method can provide very good analytical approximations for the Green's function [31,32] and exact Green's function for piecewise constant potentials [33], thus showing the versatility and generality of the approach developed in this work. These and related issues will be reported in future works [34].

This work was partially supported by the Brazilian agencies CNPq (Grant No. 313274/2017-7), Fundação Araucária (Grant No. 09/2017)), and Instituto Nacional de Ciência e Tecnologia de Informação Quântica (INCT-IQ). F.M.A. thanks Dionisio Bazeia for critical reading of the manuscript and thanks UCL CSQ group for hospitality where part of this work was done. 
[1] L. Pauling, J. Chem. Phys. 4, 673 (1936).

[2] K. Ruedenberg and C. W. Scherr, J. Chem. Phys. 21, 1565 (1953).

[3] J.-P. Roth, C. R. Acad. Sci. Paris Sér. I Math. 296, 793 (1983).

[4] T. Kottos and U. Smilansky, Phys. Rev. Lett. 79, 4794 (1997).

[5] T. Kottos and U. Smilansky, Ann. Phys. (N.Y.) 274, 76 (1999).

[6] H. Schanz and U. Smilansky, Phys. Rev. Lett. 84, 1427 (2000).

[7] A. G. M. Schmidt, B. K. Cheng, and M. G. E. da Luz, J. Phys. A 36, L545 (2003).

[8] F. M. Andrade, A. G. M. Schmidt, E. Vicentini, B. K. Cheng, and M. G. E. da Luz, Phys. Rep. 647, 1 (2016).

[9] P. Kuchment, Proc. Symp. Pure. Math. 77, 291 (2008); arXiv:0802.3442.

[10] G. Berkolaiko and P. Kuchment, Introduction to Quantum Graphs (American Mathematical Society, Providence, RI, 2012).

[11] G. Berkolaiko, arXiv:1603.07356.

[12] J. Bolte and S. Endres, Ann. Henri Poincaré 10, 189 (2009).

[13] V. Kostrykin, J. Potthoff, and R. Schrader, Contemp. Math. 447, 175 (2007).

[14] R. Diestel, Graph Theory, 4th ed., Graduate Texts in Mathematics Vol. 173 (Springer, New York, 2010).

[15] V. Kostrykin and R. Schrader, J. Phys. A 32, 595 (1999).

[16] K. Chadan and P. C. Sabatier, Inverse Problems in Quantum Scattering Theory, 2nd ed. (Springer, New York, 1989).

[17] V. Kostrykin and R. Schrader, Contemp. Math. 415, 201 (2006).

[18] Given two matrices $M$ and $N$ of the same order, the SchurHadamard product is defined by $(M \circ N)_{i j}:=(M)_{i j}(N)_{i j}$.
[19] E. N. Economou, Green's Functions In Quantum Physics, 3rd ed. (Springer-Verlag, Berlin, 2006).

[20] D. Waltner and U. Smilansky, Acta Phys. Pol. A 124, 1087 (2013).

[21] E. Ragoucy, J. Phys. A 42, 295205 (2009).

[22] V. Caudrelier and E. Ragoucy, Nucl. Phys. B 828, 515 (2010).

[23] S. Gnutzmann and U. Smilansky, Adv. Phys. 55, 527 (2006).

[24] In other words, we label our vertices in such a way that the entrance vertex is labeled 1 and the exit vertex is labeled $n$.

[25] C. R. J. Roger A. Horn, Matrix Analysis (Cambridge University Press, Cambridge, UK, 2012).

[26] V. Futorny, R. A. Horn, and V. V. Sergeichuk, Linear Algebra Appl. 519, 278 (2017).

[27] For example, for $n=2$, three words need to be checked $w(s, t)=s ; s^{2}$ and $s t$, and for $n=3$, seven words need to be checked $w(s, t)=s ; s^{2}, s t ; s^{3}, s^{2} t ; s^{2} t^{2} ;$ and $s^{2} t^{2} s t$.

[28] F. M. Andrade and M. G. E. da Luz, Phys. Rev. A 84, 042343 (2011).

[29] R. Blümel, Y. Dabaghian, and R. V. Jensen, Phys. Rev. Lett. 88, 044101 (2002).

[30] R. Blümel, Y. Dabaghian, and R. V. Jensen, Phys. Rev. E 65, 046222 (2002).

[31] M. G. E. da Luz, B. K. Cheng, and M. W. Beims, J. Phys. A 34, 5041 (2001).

[32] F. M. Andrade, B. K. Cheng, M. W. Beims, and M. G. E. da Luz, J. Phys. A 36, 227 (2002).

[33] F. M. Andrade, Phys. Lett. A 378, 1461 (2014).

[34] F. M. Andrade and S. Severini (unpublished). 